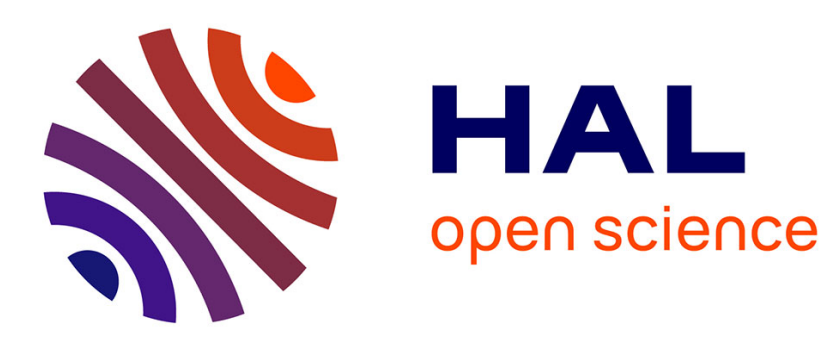

\title{
Étude de l'aimantation des corps ferromagnétiques au-dessus du point de Curie
}

\author{
Pierre Weiss, G. Foex
}

\section{To cite this version:}

Pierre Weiss, G. Foex. Étude de l'aimantation des corps ferromagnétiques au-dessus du point de Curie. J. Phys. Theor. Appl., 1911, 1 (1), pp.274-287. 10.1051/jphystap:0191100104027401 . jpa-00241669

\section{HAL Id: jpa-00241669 https://hal.science/jpa-00241669}

Submitted on 1 Jan 1911

HAL is a multi-disciplinary open access archive for the deposit and dissemination of scientific research documents, whether they are published or not. The documents may come from teaching and research institutions in France or abroad, or from public or private research centers.
L'archive ouverte pluridisciplinaire HAL, est destinée au dépôt et à la diffusion de documents scientifiques de niveau recherche, publiés ou non, émanant des établissements d'enseignement et de recherche français ou étrangers, des laboratoires publics ou privés. 


\section{ÉTUDE DE L'AIMANTATION DES CORPS FERROMAGNÉTIQUES AU-DESSUS DU POINT DE CURIE ${ }^{(1)}$ :}

Par Pierre Weiss et G. FOEX.

L'hypothèse du champ moléculaire $\left(^{2}\right)$ a donné le moyen de déduire de la théorie cinétique du paramagnétisme la loi de variation thermique de l'aimantation à saturation. Cette loi a été soumise au contrôle de l'expérience et a été, dans quelques cas, trouvée en accord frappant avec elle. L'état actuel de cette vérification, pour laquelle de nouvelles expériences sont en voie d'exécution, a été résumé dans l'introduction d'un mémoire récent $\left({ }^{3}\right)$

La même théórie a donné un résultat particulièrement simple relatif aux propriétés des ferromagnétiques aux températures au-dessus de celle de la disparition du ferromagnétisme spontané, ou, comme nous dirons dorénavant, au-dessus du point de Curie. Au-dessus de ce point, le coefficient d'aimantation spécifique \% (susceptibilité rap-

(1) Communication faite à la Société française de Physique, par M. Foex : séance du 3 mars 1911.

(2) J. de Phys., $4^{\circ}$ s., t. VI, p. 661: 1907.

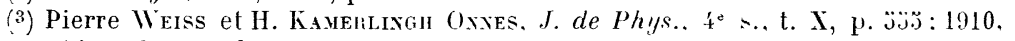
et Archives des sc. pleys. et nal.. t. XXX. p. 3il et th?: 1910. 
AIMANTATION DES CORPS FERROMAGNETIQLES 2:.: portée à l'unité de masse multiplié par l'excès de la température T sur celle 1 du point de Curie $\Theta$ est égal à une constante C qui n'est autre que la constante de Curie qu'aurait la substance si. par la suppression des actions mutuelles entre les molécules, elle était devenue paramagnétique $\left({ }^{2}\right)$ :

$$
\%(\mathbf{T}-\Theta)=\mathrm{C} \text {. }
$$

Dans le mémoire cité ci-dessus une première vérification tirée des expériences de Curie sur le fer a été indiquée. La variation hyperbolique de la susceptibilité en fonction de la température $\mathrm{T}-\Theta$, qui avait échappé à Curie, est exactement réalisée. La même remarque aurait pu ètre faite à propos des expériences de Curie sur le nickel et la magnétite, considérées dans des intervalles de température relativement étendus.

L'intérêt de la détermination de la constante C de l'équation (1) tient à la manière directe dont sa connaissance est liée à celle du champ moléculaire. L'équation :

$$
\mathrm{H}_{\mu 2}=\mathrm{XI},
$$

qui est l'expression de l'hypothèse fondamentale, introduit la proportionnalité du champ moléculaire $\mathrm{H}_{m}$ à l'intensité d'aimantation I au moyen du coefficient $\mathrm{N}$, lequel figure aussi dans la relation donnée par la théorie :

$$
\text { C.N.D }-\Theta,
$$

où $\mathrm{D}$ représente la densité.

Méthode de mesure. - La méthode de mesure employée dérive du même principe que celle dont Curie s'est servi dans son Étude rles propriétés magnétiques des corps à diverses températures $\left({ }^{3}\right)$. Elle consiste à déduire le coefficient d'aimantation spécifique /. d'un corps de masse $m$ de la force $m / \mathrm{H} \frac{\text { iII }}{\lambda, s^{\prime}}$ qu'exerce sur lui, suivant la direction des $x$, un champ magnétique non uniforme $H$. On dispose l'expérience de façon que cette force ait un maximum en un certain point du champ. En plaçant le corps en ce point on s’affranchit de la cause

(1) J. de Phyx., 4 $4^{\circ}$ série, t. VI, p. 68. : 1907.

(2) Nous désignons par $\mathrm{T}$ et $\Theta$ les températures absolues, par $t$ et 0 relles de l'échelle ordinaire.

(3) P. Curie, Ann. Chim. Phys.. ís., t. V, p. 289 (1893; : OEıvres, 1. 232. 
d'erreur consistant dans les petites variations de la position relative du corps et de l'aimant.

Pour déduire $\%$ de cette force on peut, comme l'a fait Curie, mesurer $\mathrm{H}$ et $\frac{\partial H}{\partial x}$ en valeur absolue. Mais il est plus commode de procéder par comparaison et d'amener au maximum d'attraction une masse connue d'un corps de coefficient d'aimantation connu et de mesurer la force que l'aimant exerce sur lui. Le rapport des deux forces donnera le rapport des moments magnétiques des deux corps et par suite le rapport des coefficients d'aimantation.

C'est à cette dernière méthode que nous nous sommes arrêtés. Elle exige deux séries d'opérations entièrement distinctes :

$1^{\circ}$ La mesure en valeur absolue du coefficient d'aimantation de corps types ;

$2^{\circ}$ Les mesures proprement dites sur les diverses substances ferromagnétiques.

\section{I. - MESURE DE COEfficients D'Ainantation de CORPS Types.}

Nous avons déterminé les coefficients d'aimantation de deux solutions de nitrate de nickel et d'une solution de nitrate de cobalt par la méthode bien connue de l'ascension du liquide dans la branche d'un tube communiquant placée dans un champ magnétique, qui a

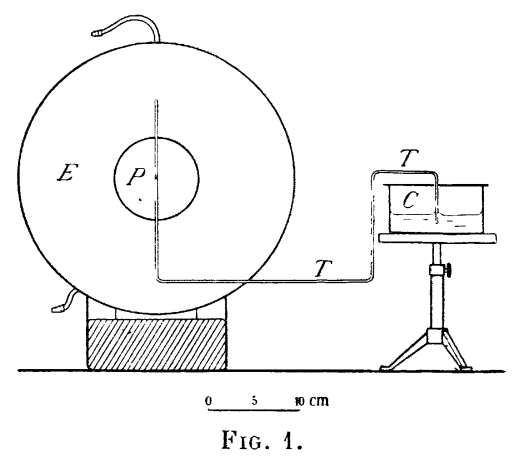

été imaginée par Quincke. Les appareils sont représentés dans la $f g .1$. Un cristallisoir de 10 centimètres de diamètre contient la solution. Il en part un tube de 2 millimètres de diamètre intérieur qui, d'abord recourbé en siphon, se termine par une branche verti- 
AIMANTATON DES CORPS FERROMAGX́tIQUES git cale, placée dans le champ d'un électro-aimant. Ce champ, obtenu avec des pièces polaires planes de 9 centimètres de diamètre, écartées de 7 millimètres, est sensiblement uniforme dans une étendue de 3 à 4 centimètres de diamètre.

On s'arrange de façon que le niveau du liquide soit au centre du champ quand l'aimant est excité. On mesure la dénivellation qui se produit au moment de l'établissement du champ au moyen du cathétomètre, ce qui est rendu facile par la forte coloration des liquides.

Soient $\%$ le coefficient d'aimantation du liquide:

$\mathrm{H}$ et $\mathrm{H}^{\prime}$, les champs magnétiques à la surface du liquide dans le tube et dans le cristallisoir;

$s$ et $\mathrm{S}$, les sections de ceux-ci;

ò, la dénivellation, on a :

$$
\%\left(\mathbf{H}^{2}-\mathbf{H}^{\prime 2}\right)=2 \dot{\delta} \frac{\mathrm{S}+s}{\mathrm{~S}} g .
$$

Ici $(\mathrm{S}+s): \mathrm{S}=1,00$ ห, rapport très voisin de l'unité. De plus le cristallisoir est assez éloigné de l'aimant pour que $1 \mathrm{I}^{\prime 2}$ soit tout à fait négligeable vis-à-vis de $\mathrm{H}^{2}$. L'intensité $\mathrm{H}$ était voisine de 16.000 gauss, alors que $H^{\prime}$ ne valait que 20 gauss.

On obtient ainsi le coefficient d'aimantation apparent dans l'air. C'est aussi celui qui intervient dans l'emploi qui sera fait de ces solutions. La correction à laire pour ramener \% au vide serait inférieure à $1 / 200$ de sa valeur.

Solutions. - Le nitrate de nickel et le nitrate de cobalt ont été préférés à d'autres sels parce qu'ils joignent à une grande solubilité un coefficient d'aimantation assez élevé. De plus ils sont inaltérables à l'air et peuvent se conserver longtemps en solution. Ces sels purs (Kahlbaum) ont été dissous dans de l'eau distillée en quantités telles que les solutions, tout en étant concentrées, n’abandonnassent pas de cristaux par refroidissement de quelques degrés. Les mesures ont toujours été faites en opérant sur toute la solution à la fois $(200$ à 300 centimètres cubss).

Tube ì ascension. - Le tube a été calibré sur une certaine longueur par la méthode de la goutte de mercure. On a pu trouver de cette façon une région longue de $2^{\prime m}$, ö où le diamètre du tube est très constant. C'est dans cette région que nous avons opéré pour éviter les erreurs provenant des variations de l'ascension capillaire.

Tous les ustensiles en verre ont été lavés à l'acide chromique, à J. de Phys., :̈* série, t. I. (Avril 1911.) 
la potasse, puis rincés avec de l'eau et avec de la solution elle-mème. A chaque expérience le tube était mouillé en aspirant la solution jusqu'au-dessus du niveau auquel elle s'arrètait, quand l'aimant était excité.

Champ magnétique. - Pour se mettre à l'abri d'une influence possible de l'aimantation résiduelle de l'électro-aimant, on a pris soin de faire toutes les mesures du champ ainsi que celles de l'ascension du liquide pour deux valeurs égales et de signe contraire du courant d'excitation.

Les mesures du champ ont été faites avec la balance magnétique absolue de M. A. Cotton, dont l'élément de courant inférieur était placé au centre de la région uniforme du champ. La correction due à l'élément de courant supérieur a été faite. Les courants envoyés dans la balance ont été mesurés avec un ampèremètre Siemens et Halske, comparé avec un autré ampèremètre étalonné à l'aide d'un élément Weston d'un ohm contrôlé à l'Institut physico-technique de Charlottenburg et du potentiomètre.

Résultats (tous les coefficients d'aimantation sont ramenés à la température de $13^{\circ}$ au moyen de la loi de Curie):

Solution $n^{\circ} 1$ de nitrate de nickel.

Champ pour un courant de 15 ampères, 1390 i gauss.

$\begin{array}{ccc}\text { Courant } & \begin{array}{c}\text { Hauteur } \\ \text { lue au cathétomètre }\end{array} & \text { Ascension } \\ 0 & 389,05 & \\ +13 \text { amp. } & 601,2: 3 & 12,20 \mathrm{~mm} . \\ 0 & 589,0.3 & \\ -13 & 601,23 & 12,20 \\ 0 & \because 389,03 & \\ +13 & 601,23 & 12,20 \\ & \%=9,45.10^{-6} & \end{array}$

Champ pour un courant de 20 ampères, 16810 gauss.

\begin{tabular}{|c|c|c|}
\hline Courant & $\begin{array}{c}\text { Ilauteur } \\
\text { lue au cathétometre }\end{array}$ & Ascension \\
\hline 0 & :89,0̈ & \\
\hline - $20 \mathrm{amp}$. & 602,60 & $13,3.0 \mathrm{~mm}$. \\
\hline 0 & $589,0$. & \\
\hline-20 & 602,60 & 13,3.; \\
\hline 0 & :89,0 & \\
\hline$\div 20$ & $602,6 . ;$ & 13,60 \\
\hline 0 & 589,03 & \\
\hline-20 & $\begin{aligned} & 602,63 \\
\gamma= & 9,+3.10^{6}\end{aligned}$ & 13,60 \\
\hline
\end{tabular}


AIMANTATION DES CORPS FERROMAGNETIQLES :2

En moyenne :

$$
x=9,4^{\prime} \cdot 10^{-6}
$$

Solution $n^{0} 2$ de nitrate de nickel.

(autre électro-aimant;

Clıamp pour un courant de 15 ampères, 16080 gauss.

\begin{tabular}{|c|c|c|}
\hline Température & Ascension & Coeff. d'aimant à 180 \\
\hline $16^{\circ}, 0$ & $\tau, 85 \mathrm{~mm}$. & 6.030 - $10^{-6}$ \\
\hline $16^{\circ}, 0$ & $7.8 . j$ & \\
\hline $13^{\circ}, 2$ & $\tau, 90$ & \\
\hline $13^{\circ}, 2$ & $7,9: i$ & $601 \quad 10^{-6}$ \\
\hline $13^{\circ}, 0$ & $\tau, 90$ & $0,01 \cdot 10$ \\
\hline $13^{\circ}, 0$ & $7,9 \ddot{\jmath}$ & \\
\hline
\end{tabular}

Champ pour un courant de 20 ampères, 16910 gauss.

$\left.\begin{array}{cl}\text { Température } & \text { Ascension } \\ 12^{\circ}, 2 & 8,70 \mathrm{~mm} . \\ 12^{\circ}, 2 & 8, \pi 30\end{array}\right\} \begin{gathered}\text { Loelf. d'aimant à } 13^{\circ} \\ \because, 9 \tau \cdot 10^{-6}\end{gathered}$

En moyenne $\%=6,01 \cdot 10^{-6}$ pour la solution $\mathrm{n}^{\circ} 2$ de nitrate de nickel.

Solution $n^{0} 3$ de nitrate de cobalt, $\mathrm{H}=16080$.

$\begin{array}{ccc}\text { Température } & \text { Ascension } & \text { Coeff. daimant à } 13^{\circ} \\ 12^{\circ}, 5 & 19,00 & 1+40 \cdot 10^{-6} \\ 13^{\circ}, 0 & 18,9: 3 & 14,39 \cdot 10^{-6}\end{array}$

En moyenne \% $=14,40 \cdot 10^{-6}$.

Pour avoir une vérification, nous avons comparé les diverses solutions entre elles dans l'appareil de mesure des coefficients d'aimantation qui sera décrit plus loin. L'appareil, étalonné au moyen de la première ampoule contenant la solution $n^{\circ} 2$, a servi à déterminer, par plusieurs mesures dont on a pris la moyenne, les nombres de l'avant-dernière colonne. Ceux de la dernière sont les résultats de la méthode d'ascension.

\begin{tabular}{|c|c|c|c|c|}
\hline \multirow{2}{*}{ Solution } & \multirow{2}{*}{ Nasse } & Cinurant & \multicolumn{2}{|c|}{ Coeff. daimantation troure } \\
\hline & & $\begin{array}{l}\text { de } \\
\text { rompensation }\end{array}$ & par comparaison & directement \\
\hline No 2 & $0.1810 \mathrm{gr}$. & 0,8638 amp. & - & $6,01 \cdot 10^{-6}$ \\
\hline $\mathrm{N}^{0} \underset{\sim}{2}$ & $0, \because 2.29$ & $2, .000$ & $0,03 \cdot 10$ & $6,01 \cdot 10^{-6}$ \\
\hline$x=1$ & $0,631: 2$ & 4,96 & $9, \ddot{9} 9.10$ & $9, \mathbf{y}^{\prime} \cdot 10^{-6}$ \\
\hline Yo 3 & 0,13 往 & 1,811 & $1 ;, \pm 8.10$ & $1+40 \cdot 10^{-6}$ \\
\hline
\end{tabular}

Les nombres de l'avant-dernière colonne concordent avec ceux de la dernière dans la mesure où l'on pouvait l'attendre pour des obser- 
vations en général exactes à moins de 10,0 près. La divergence un peu plus grande de la troisième ligne provient sans doute d'erreurs notables dans la température.

La petitesse de l'influence de l'air est l'un des avantages de l'étalonnement fait avec des substances de grand coefficient d'aimantation spécifique. Cet avantage s'accentue encore pour les substances de forte densité sur lesquelles ont porté les mesures définitives. De même la correction du magnétisme du support est souvent tout à fait insensible.

Coefficient d'aimantation d'une substance étalon. - Nous avons saisi l'occasion offerte par notre appareil étalonné en valeur absolue pour déterminer le coefficient d'aimantation d'une substance type pouvant servir de repère dans des mesures magnétiques ultérieures. Le sulfate de cobalt nous a paru satisfaire à toutes les conditions que doit remplir une telle substance : il a une susceptibilité élevée (plus élevée que les sels de nickel), il est facile à obtenir suffisamment pur et ne s'altère pas comme les sels de fer. Nous avons fait deux séries de mesures sur ce sel à l'état solide. L'une a porté sur le sel anhydre, l'autre sur le sel cristallisé à sept molécules d'eau.

Le sulfate de cobalt anhydre a été obtenu en chauffant le sel (pur, Kahlbaum) avec de l'acide sulfurique jusqu'à ce que les vapeurs blanches caractéristiques de l'acide sulfurique aient complètement cessé de se produire. La poudre ainsi préparée a été enfermée encore toute chaude dans une ampoule de verre qui a été immédiatement scellée à la lampe et qui a servi aux mesures.

La constante de l'appareil, pour un champ produit par un courant de 1.500 ampères, a été déterminée au moyen des deux ampoules contenant la solution $n^{\circ} 2$.

Le coefficient d'aimantation est donné par la formule (1):

$$
\%=1,220 \cdot 10^{-6} \frac{\mathrm{I}}{\mathrm{m}} \text {. }
$$

Avec un premier échantillon de sulfate de cobalt nous avons trouvé :

$$
\mathrm{I}=\tau, 96 \text { ampères pour } m=0 ", 167 ;,
$$

et par suite:

$$
\%=58,3 \cdot 10^{-6} \text { à } 20^{\circ} \text {. }
$$

(1) Voir p. 282 du présent mémoire. 
Une deuxième préparation a donné :

$$
\mathrm{I}=: 3,92 \text { ampères pour } m=02.1232
$$

et

$$
\%=\check{3} 8,9 \cdot 10^{-6} \text { à } 20^{\circ} \text {. }
$$

En moyenne:

$$
\%=.88 \cdot 10^{-6} \text {. }
$$

Le sulfate de cobalt ì 7 molécules d'eau (pur, Kahlbaum) a été réduit en poudre et enfermé dans une petite ampoule. Pour $m=1^{\mathrm{g}}, 3$ วัว on a trouvé : $\mathrm{I}=3,432$, d'où :

$$
\chi=31,9 \cdot 10^{-6} \text { à } 20^{\circ} .
$$

En multipliant ce nombre par le rapport:

$$
\frac{\mathrm{SO}^{\prime} \mathrm{Co}+7 \mathrm{H}^{2} \mathrm{O}}{\mathrm{SO} \mathrm{CoO}^{\prime}}=\frac{281}{183^{3}}
$$

on trouve :

$$
\%=: 38,0 \cdot 10^{-6} \text {, }
$$

nombre qui concorde avec la valeur trouvée directement pour le cobalt anhydre dans la limite de précision des expériences, surtout si l'on tient compte de la difficulté de se débarrasser complètement de l'eau d'interposition.

Le nombre donné par M. Meslin (1) pour le sulfate de cobalt est de $240 / 0$ plus grand que celui-là $(39,7$ au lieu de 31,9$)$. Il est difficile de savoir à quoi tient un pareil écart. Nous pensons cependant qu'il peut provenir de ce que M. Meslin rapporte ses mesures à l'eau dont le très faible coefficient d'aimantation $\left(-0.79 .10^{-6}\right)$ peut être altéré dans le sens expliquant la divergence par une très petite quantité d'impuretés magnétiques. Des différences de 15 à $170 / 0$ se retrouvent, en effet, entre les résultats de Meslin et de Stephan Meyer $\left({ }^{2}\right)$. Ce dernier, en prenant pour corps de comparaison le mercure avec $\%=-2,03 \cdot 10^{-6}$, a trouvé, pour le sulfate de

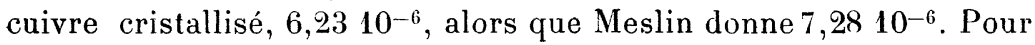
le sulfate de magnésie, l'écart est encore plus fort (Meslin, - 0,616 ; Meyer, - 0,303).

(1) Ann. Chim. Phys., 8 série. t. VII (févr. 1906).

(2) Sitz. Aliad. Wien.. t. GVIII. II juillet 1899;. 
II. - mesures sur les substances ferromagétiques.

La fig. 2 représente, vues d'en haut, les parties essentielles del'appareil pour la comparaison des coefficients d'aimantation. Les

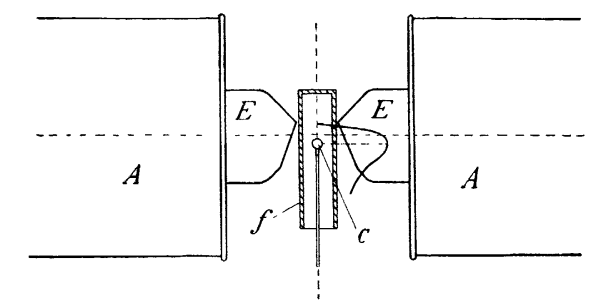

FIG. 2.

noyaux E, E de l'électro-aimant AA se terminent par des cônes qui ont été tronqués par des plans inclinés sur l'axe. On a réalisé ainsi un entrefer de même forme que celui des expériences classiques de Curie. Le champ non uniforme a pour effet d'attirer la substance $c$ vers l'axe de l'aimant. Tant que la substance a une susceptibilité indépendante du champ, la position pour laquelle la force est maximum est pratiquement invariable, quel que soit le courant. Comme le montre la courbe dessinée dans la $f$ g. 2, qui représente cette attraction en fonction de la distance à l'axe de l'aimant, ce maximum est assez plat pour que l'on puisse, sans erreur de quelque importance, employer des corps d'étendue assez différente.

La comparaison des attractions se fait par une méthode de zéro : on équilibre l'action du champ magnétique sur la substance par la répulsion électromagnétique de deux bobines coaxiales (fig. 3 ), dont l'une B est fixe et l'autre calée sur le fléau qui supporte la substance dans le champ. Le produit des courants circulant dans les deux bobines au moment où l'équilibre est établi, donne une mesure de la force et par suite du produit de la masse par le coefficient d'aimantation du corps. Soient I et $i$ les courants dans les deux bobines, in la masse, le coefticient d'aimantation sera :

$$
\%=\mathrm{B} \frac{\mathrm{I} \cdot i}{m}=\mathrm{A} \frac{\mathrm{I}}{m} \text {. }
$$

Un étalonnement de l'appareil donnera A qui est la seule cons- 
tante instrumentale à connaître, lorsque le courant dans la bobine mobile est choisi une fois pour toutes, pour une même série d'expériences.

Aimant. - Un électro-aimant Ruhmkorff $A$ pouvant supporter jusqu'à $2 \partial ّ$ ampères donne, avec l'entrefer adopté, des champs de 4 à 5.000 gauss. La plupart des expériences ont été faites avec un champ de 2.130 gauss. L'aimant est monté sur un chariot $\mathrm{D}$, muni de roulettes à gorges qui reposent sur deux rails parallèles. Une manivelle actionnant une vis permet do faire mouvoir l'aimant par rapport à la substance pour rechercher le maximum d'attraction. Un cercle divisé calé sur la vis sert à repérer la position de l'aimant. Le circuit de l'aimant comprend un ampèremètre de précision et deux rhéostats de résistance très différente montés en parallèle qui permettent un réglage très exact du courant.

Suspension de la substance dans le champ maynélique. - Le corps est couché dans une petite coupelle hémisphérique en platine, représentée en $c$ dans la $f g$. 3. Cette coupelle est portée par un tube fendu suivant deux génératrices opposées. Dans ce tube entre à frottement dur un autre tube, en silice fondue, de 30 centimètres de longueur et de 2 millimètres de diamètre. Ce tube lui-même est suspendu horizontalement dans le plan de symétrie de l'aimant et peut osciller dans ce plan.

La potence $\mathrm{K}$, fixée à un pilier en maçonnerie indépendant du plancher du laboratoire, porte le tube au moyen de quatre rubans de cuivre argenté très mince (largeur, 1 millimètre; longueur, 220 millimètres), attachés en $t, t$ aux extrémités de deux tiges transversales en laiton et qui convergent en forme de $V$, pour aller se fixer à deux anneaux de cuivre montés sur le tube.

Ce mode de suspension a de grands avantages. Il supprime entièrement les déplacements latéraux de la substance dans le champ magnétique, il est plus robuste que les appareils à torsion et, par la facilité avec laquelle il se prête à l'emploi du dispositif de compensation électro magnétique, permet de supprimer les causes d'erreur signalées par Meslin et provenant des trépidations et des changements de zéro.

Enfin, l'appareil peut être employé comme appareil à déviation à la façon d'un pendule. Il suffit pour cela de fixer un miroir sur l'une des paires de rubans de suspension. C'est avec cette disposition qu'ont été faites les expériences préliminaires. 
Dispositif de compensation. - La compensation de l'attraction de laimant sur la substance est obtenue au moyen de deux bobines coaxiales $B$ et 7 , dont l'une, calée sur le tube, pénètre dans l'autre qui est fixe. Le centre de chacune des bobines coïncide avec l'une des extrémités de l'autre de manière à diminuer le plus possible les changements de constante dus à un petit déplacement relatif.
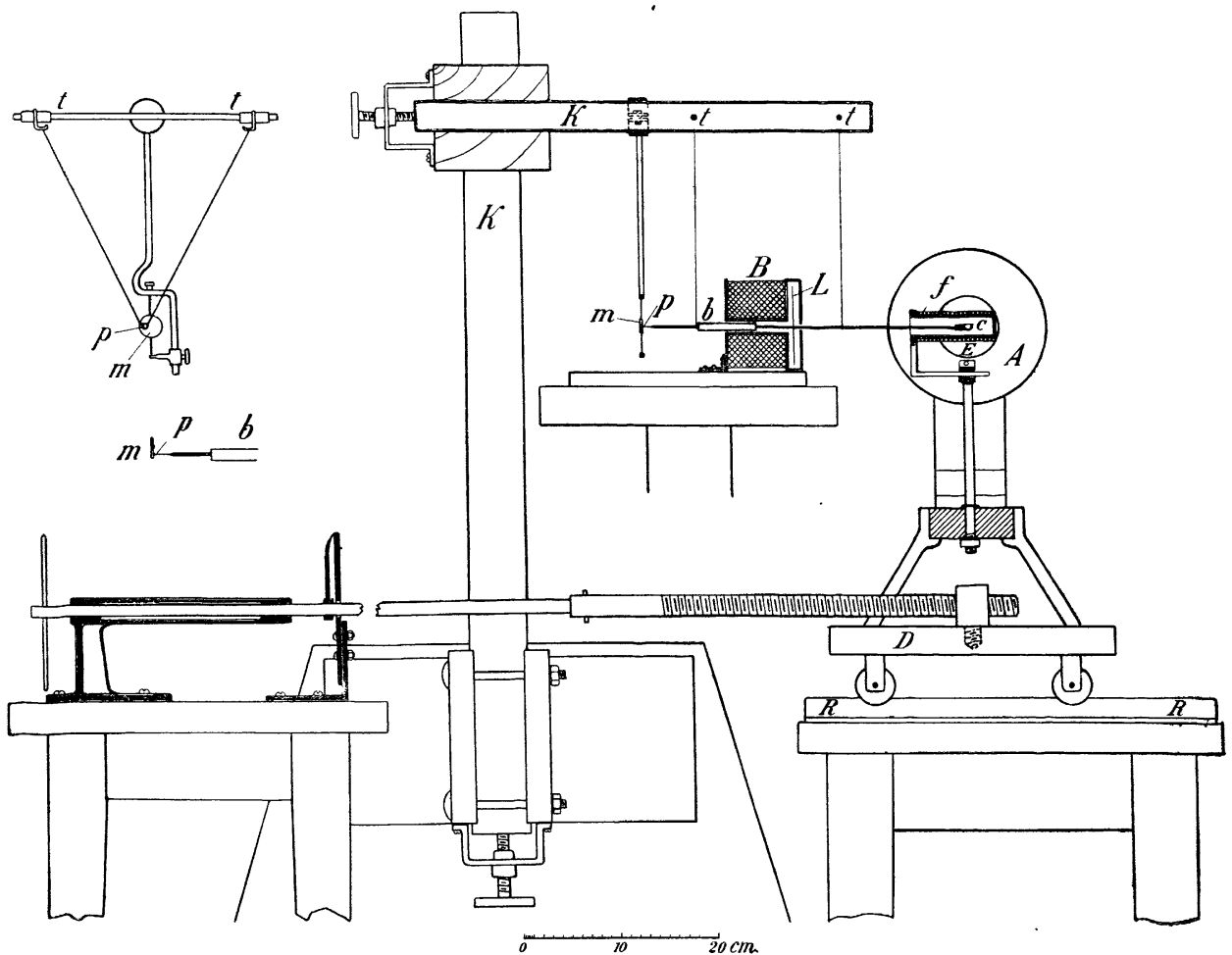

FIG. 3.

La bobine $b$ a été faite en enroulant une seule couche de fil de $0^{\mathrm{mm}}, 2 \breve{\partial}$ sur un tube de verre très mince de 7 millimètres de diamètre. Elle a 6 centimètres de long et porte 150 tours de fil. Le fil recouvert de soie qu'on avait employé d'abord présentait des traces de ferromagnétisme, se manifestant notamment par un certain magnétisme rémanent; il a été remplacé avec avantage par du fil émaillé. Le courant est amené à cette bobine par l'une des paires de fils de sus- 
AIMANTATION DES CORPS FERROMAGNÉTIQUES 28ว pension et ressort par l'autre. Le courant dans la bobine mobile a été de 0,01 à 0,02 ampère. La bobine fixe $B$ est formée de $3 \check{0} 0$ tours d'un gros fil pouvant supporter jusqu'à 1 ŏ ampères et occupant une longueur de 6 centimètres.

Appareil de lectures. - Pour repérer la position d'équilibre du tube de quartz, on l'a terminé par une pointe $p$ qui vient buter excentriquement contre un miroir $m$, porté par une lame tendue en bronze phosphoreux. La méthode ordinaire de lecture des déviations du miroir amplifie donc considérablement les déplacements du tube et donne toute la sensibilité désirable. Pour éviter les frottements entre la pointe et le miroir, on a collé sur le dos de celui-ci un morceau de lamelle de couvre-objet de microscope et l'on a arrondi en la fondant la pointe effilée qui termine le tube de silice. On obtient de cette façon une constance absolue du zéro. L'appareil est complété par un amortisseur à air L.

Obtention et mesure des températures élevées. - La substance est chauffée au moyen d'un four électrique $t$, invariablement lié à l'aimant. Il est formé d'une carcasse cylindrique en nickel, fermée à une extrémité, servant à uniformiser la température. Elle a 2 centimètres de diamètre, 9 centimètres de longueur et $0^{\mathrm{cm}}, 2$ d'épaisseur, et porte deux couches de fil de nickel de 1 millimètre, isolé à l'amiante. Le tout est noyé dans du kaolin qui sert d'isolant thermique.

Ce four s'est montré suffisant au point de vue de l'uniformité de la température, ainsi qu'il résulte du tableau suivant, résumant l'étude qui en a été faite pour une température voisine de $900^{\circ}$.

\begin{tabular}{|c|c|}
\hline Distances à la bouche & Microvolts du couple platine-platine rhodié \\
\hline $1 \mathrm{~cm}$. & ð̋80 \\
\hline 2 & 7360 \\
\hline 3 & 7 905 \\
\hline 4 & 8307 \\
\hline 5 & 8466 \\
\hline 6 & $8 \dddot{008}$ \\
\hline 7 & $8 \div 6$ \\
\hline 8 & 8268 \\
\hline $8, \ddot{3}$ & 8150 \\
\hline
\end{tabular}

10 microvolts correspondant environ à $1^{\circ}$, on peut déduire de ces nombres qu'il existe un maximum allongé de température, dans le voisinage duquel les fluctuations ne dépassent pas $2^{\circ}$ dans un intervalle de 1 centimètre. 
Dans une section perpendiculaire à l'axe du four, la constance de la température est encore plus satisfaisante:

$\begin{array}{lc}\text { Distance à la paroi supérieure } & \text { Microrolts } \\ 0 \mathrm{~mm} . & 8564 \\ 2 & 8 \% 43 \\ 4 & 8 \% 39 \\ 9 \text { axe } & 8 \% 30 \\ 16 & 8544 \\ 18 \text {, paroi inférieure) } & 8569\end{array}$

Le maximum de température sur l'axe du four se trouve exactement à $6^{\prime m}, 2$ de la bouche. C'est en ce point qu'ont été placés le corps étudié et la soudure du couple thermo-électrique. A cet effet le four a été fixé sur l'aimant de manière à faire coïncider le maximum d'attraction avec le maximum de température.

Ce four n'a pas d'action sensible sur le champ magnétique : les deux couches de fil de nickel sont enroulées en sens contraire et, d'autre part, aux températures où l'on opère, le tube de nickel a perdu ses propriétés fortement magnétiques.

La mesure des températures se fait au moyen d'un couple platineplatine rhodié qui a été étalonné plusieurs fois à l'aide des poin ts fixes de Hoborn et Day. Les forces électromotrices de ce couple sont mesurées au potentiomètre, en prenant comme élément de comparaison un weston.

Étalonnement. - L'appareil a été étalonné au moyen de deux ampoules différentes contenant de la solution $\mathrm{n}^{\circ} 2$ de nitrate de nickel dont la susceptibilité avait été déterminée avec le plus de soin.

L'électro-aimant n'est pas très éloigné de la bobine mobỉe. Mème lorsqu'on prend la précaution de n'y faire passer que des courańts de faible intensité, la force qu'elle éprouve directement de la part de l'aimant est comparable à celle qu'il exerce sur la substance. On s'en affranchit en faisant deux observations dans lesquelles le courant de la bobine mobile garde la même valeur et pour lesquelles le sens du courant est changé dans les deux bobines à la fois. Soient $I_{1}$ et $I_{2}$ les deux valeurs du courant dans la bobine fixe, leur somme I est introduite dans les calculs. La valeur constante du courant dans la bobine mobile a été 0,01980 ampère.

Solution $n^{\circ} 2$ de nitrate de nickel :

$$
z=6,01 \cdot 10^{-6} \text { à } 13^{\circ} \text {, temp. } 20^{\circ} \text {. }
$$


AIMANTATION DES CORPS FERROMAGNÉTIQLE- Q2:Ampoule contenant $0^{\mathrm{g}}, 1810$ de solution :

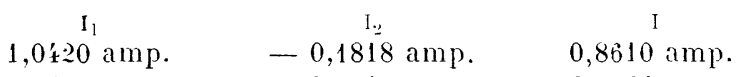

$$
\begin{aligned}
& 1,0 \mathrm{t} 2.5-0,1761 \quad 0866 \mathrm{t} \\
& 1,0341-0,1696 \quad 0.86 \% .: \\
& 1,03 \text { อั1 }-0,1 \text { iा8 } \\
& \text { Moyenne : } \overline{0,8638}
\end{aligned}
$$

La constante de l'appareil, c'est-à-dire le nombre par lequel il faut multiplier I pour obtenir le coefficient d'aimantation, est :

$$
\mathrm{A}=1,229 \cdot 10^{-6}
$$

Même solution, ampoule contenant $0^{\circ}, 522\left(\right.$ temp. $20^{\circ}$ ):

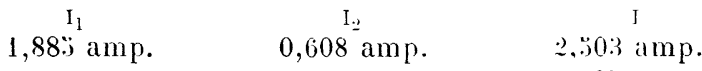

$$
\begin{aligned}
& 1,922=0,: 338 \quad 2,303 \\
& \begin{array}{lll}
1,916 & 0, . ; 8: 3 & 2,498
\end{array} \\
& \text { Moyenne : } \overline{2,302} \\
& \mathrm{~A}=1,223 \cdot 10^{-6} \text {. }
\end{aligned}
$$

Moyenne :

$$
\mathrm{A}=1,226 \cdot 10^{-6} .
$$

A chaque nouvelle substance, l'appareil a été réétalonné au moyen de l'ampoule de $0^{\frac{x}{}}, \ddot{\partial} 22$ de solution $\mathrm{n}^{0} 2$. Les résultats ont toujours été assez voisins de celui du premier étalonnement.

Les nombres qui précèdent montrent que l'on peut faire varier dans d'assez larges limites la quantité de substance employée, grâce à la souplesse du dispositif de compensation électrodynamique, qui permet de mesurer des forces de grandeurs très différentes. Ils montrent aussi que la région d'attraction maxima est suffisamment étendue pour qu'il ne résulte aucune erreur appréciable de l'emploi de corps de volumes très divers.

Les mesures proprement dites ont été faites de la même manière que les étalonnements. Un certain nombre de renseignements complémentaires sur des variantes expérimentales seront données au fur et à mesure de la description des résultats qui fera l'objet d'un autre article. 